Article

\title{
Thermally Healable and Recyclable Graphene-Nanoplate/Epoxy Composites Via an In-Situ Diels-Alder Reaction on the Graphene-Nanoplate Surface
}

\author{
Cho-Rong Oh ${ }^{1,+}$, Dae-Il Lee ${ }^{1,+}$, Jun-Hong Park ${ }^{2, *}$ and Dai-Soo Lee ${ }^{1, * \text { (D) }}$ \\ 1 Division of Semiconductor and Chemical Engineering, Chonbuk National University, Baekjedaero 567, \\ Jeonju 54896, Korea; ohcho38@naver.com (C.-R.O.); ldi4084@naver.com (D.-I.L.) \\ 2 R \& D Center, Lotte Advanced Materials, Sandan-ro 334-27, Yeosu 59616, Korea \\ * Correspondence: jh711.park@lottechem.com (J.-H.P.); daisoolee@jbnu.ac.kr (D.-S.L.); \\ Tel.: +82-61-689-1731 (J.-H.P.); +82-63-270-2310 (D.-S.L.) \\ + These authors contributed equally to this work.
}

Received: 22 March 2019; Accepted: 14 June 2019; Published: 18 June 2019

check for updates

\begin{abstract}
In this work, thermally healable graphene-nanoplate/epoxy (GNP/EP) nanocomposites were investigated. GNPs were used as reinforcement and crosslinking platforms for the diglycidyl ether of bisphenol A-based epoxy resin (DGEBA) through the Diels-Alder (DA) reaction with furfurylamine (FA). The GNPs and FA could then be used as a derivative of diene and dienophile in the DA reaction. It was expected that the combination of GNPs and FA in DGEBA would produce composites based on the interfacial properties of the components. We confirmed the DA reaction of GNPs and FA at the interface during curing of the GNP/EP nanocomposites. This procedure is simple and solvent-free. DA and retro DA reactions of the obtained composites were demonstrated, and the thermal healing properties were evaluated. The behavior of the GNP/EP nanocomposites in the DA reaction is similar to that of thermosetting polymers at low temperatures due to crosslinking by the DA reaction, and the nanocomposites can be recycled by a retro DA reaction at high temperatures.
\end{abstract}

Keywords: graphene-nanoplate; Diels-Alder reaction; epoxy composites; thermally healing

\section{Introduction}

Thermosetting polymers are widely used because of their good thermal stability, chemical resistance, and high mechanical strength. However, the reprocessing or recycling of thermosetting polymers is limited due to their molecular network architecture. Recently, numerous researchers reported self-healing and thermally remendable network polymers based on the dynamic covalent bonds formed via the Diels-Alder (DA) reaction, the metathesis of disulfide or imine, and the ester exchange reaction [1-4]. The DA reaction is a cycloaddition reaction between diene and dienophile, and the adduct is reversible at temperatures above $90^{\circ} \mathrm{C}$, as shown in Scheme 1.

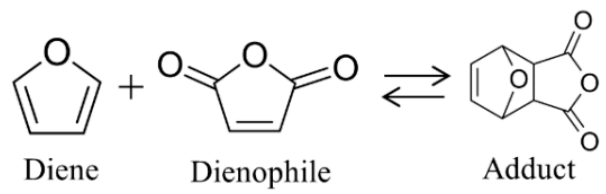

Scheme 1. The Diels-Alder reaction between diene and dienophile.

Generally, a furfuryl group is used as the diene, whereas a maleimide group is used as the dienophile based on various potential modifications. The side reaction of maleimide can continue 
when the system is exposed to high temperatures in the presence of primary or secondary amine [5-7]. In most studies, two methods are used to introduce the DA reaction in an epoxy resin. First, to introduce the DA reaction in the epoxy backbone, the synthesis of epoxy or amine-terminated DA adduct is needed to protect the double bond of dienophile [8-10]. Second, a furan-terminated backbone polymer is prepared and then reacted with bismaleimide as a crosslinker in a solvent or using extrusion [11-17]. If the dienophile could be replaced with a material other than maleic anhydride or maleimide, then epoxy composites could be prepared by the DA reaction using a simple process.

Graphite is a commonly available material and is composed of multiple layers of graphene, $s p^{2}$ bonded carbon atoms arranged in a hexagonal, or honeycomb lattice. Graphene fabricated through various exfoliation methods [18,19], has exceptional electrical, thermal, and mechanical properties, and allows for a substantial enhancement of polymer properties at a much lower content than conventional fillers [20,21]. Graphitic fillers are used to impart self-healing properties based on $\pi-\pi$ interactions or surface functionalization [22,23]. Furthermore, graphene can be used as a diene and dienophile in the DA reaction without modification. Sarkar et al recently proposed the use of single-layer graphene in DA and retro DA reactions as a dienophile or diene with another reactant at various temperatures [24]. Several studies reported the successful preparation of graphene nanoplates (GNPs), i.e., multilayered graphene, via the DA reaction of TCNE and graphite, and synthesized graphene oxide through a DA reaction between GNPs and maleic anhydride $[25,26]$. The DA reaction between a carbon-based material and mono-molecules, as well as polymers, has also been investigated $[27,28]$. Bai et al reported the synthesis of crosslinked poly(styrene-b-butadiene-b-styrene) (SBS) via the DA reaction between C60 and furan-modified SBS and evaluated its thermal healing properties [29]. Kötteritzsch and colleagues converted poly(lauryl methacrylate) using anthracene moieties in the side chain with C60 and phenyl-C61-butyric acid methyl ester, thereby producing a self-healing polymeric material [30]. According to these studies, GNPs can replace maleic anhydride or maleimide in a DA reaction. Moreover, the physical properties and electrical conductivity of the composites can be enhanced due to the characteristics of GNPs.

In the above-mentioned studies, a solvent was used for the dispersion of the GNPs and synthesis of the DA adduct. However, solvent-casting methods are not effective because the mechanical properties of the material are degraded during the drying process. This method also has issues with volatile organic compounds during film formation by drying. To address the low dispersibility of GNPs, we employed a masterbatch method in which the GNPs are dispersed in a base resin [31,32].

To simplify the surface modification process for GNPs, an in-situ DA reaction should occur during curing of the epoxy resin to produce a crosslinked GNP/epoxy (GNP/EP) nanocomposite via a DA reaction. The interfacial tension is used to determine the interfacial wetting. To ensure contact between the GNPs and the reactant for the DA reaction, the interfacial tension between the GNPs and the reactant should be lower than the sum of the interfacial tension between the matrix resin and the reactant and between the GNPs and the matrix. The interfacial tension can be calculated from the surface tension of each material. For this purpose, the commonly used Wu and Owen-Went interfacial tension equations are applied, as given in Equations (1) and (2), respectively. These formulas consider the dispersion and polar components of interfacial tension in accordance with the modified form of Fowke's equations [33-36] as follows:

$$
\begin{gathered}
\gamma_{S-l}=\gamma_{S}+\gamma_{l}-\frac{4 \gamma_{S}^{d} \gamma_{l}^{d}}{\gamma_{S}^{d}+\gamma_{l}^{d}}-\frac{4 \gamma_{S}^{p} \gamma_{l}^{p}}{\gamma_{S}^{p}+\gamma_{l}^{p}} \\
\gamma_{S-l}=\gamma_{S}+\gamma_{l}-2\left(\gamma_{s}^{d} \gamma_{l}^{d}\right)^{\frac{1}{2}}-2\left(\gamma_{S}^{p} \gamma_{l}^{p}\right)^{\frac{1}{2}} \\
\gamma_{i}=\gamma_{i}^{d}+\gamma_{i}^{p}
\end{gathered}
$$

where $\gamma_{S-l}$ is the interfacial tension between solid (s) and liquid $(l) ; \gamma_{i}$ is the surface energy of materials (i); and $\gamma_{i}^{d}$ and $\gamma_{i}^{p}$ are the dispersive and polar components of $\gamma_{i}$, respectively. 
In this study, a masterbatch was prepared by concentrating GNPs in a base resin to ensure a good dispersibility of the GNPs. The interfacial tension of each material was calculated to confirm that the GNPs would come into contact with furfurylamine (FA) in the diglycidyl ether of bisphenol A-based epoxy resin (DGEBA) to enable the DA reaction of GNPs without solvent. The mechanical properties and chemical resistance of the obtained composites were enhanced through the crosslinking effect by the DA reaction. In addition, we demonstrated that the designed system can manufacture self-healing and recyclable epoxy composites through DA and retro DA reactions.

\section{Experimental Section}

\subsection{Materials}

DGEBA and 1,6-hexanediol diglycidyl ether (HDE) were supplied by Kukdo Chemical (Seoul, Korea). Epoxy equivalent weights of DGEBA and HDE were 187 and 115 g/eq respectively. Cyclohexylamine (CA), FA, and diethylenetriamine (DETA) purchased from Sigma-Aldrich (Young-in, Korea) were reagent grades (>99\%). Dimethylformamide (DMF) and dimethyl acetamide (DMAc) were purchased from SK Chemical (Seongnam, Korea), and expandable graphite (EG) was purchased from Braide Graphite Group (Qingdao, China).

\subsection{Preparation of the GNP Masterbatch}

A 300-mL quartz cup containing $0.1 \mathrm{~g}$ of EG was placed at the center of a rotating disk in a conventional microwave oven (700 W, LG Electronics, Seoul, Korea). After 5 min of nitrogen purging, the EG was irradiated by microwave radiation for $90 \mathrm{~s}$. A total of $1 \mathrm{~g}$ of EG and $1 \mathrm{~L}$ of DMF were mixed in a 2-L beaker, and the mixture was sonicated for 1 day. After sonication, the mixture was centrifugated at $3000 \mathrm{rpm}$ for $45 \mathrm{~min}$ [37]. The supernatant obtained after centrifugation and $100 \mathrm{~g}$ of base resin were mixed in a round-bottom flask and magnetically stirred for $1 \mathrm{~h}$. Concentrated GNPs in epoxy resin were formed after the removal of DMF at $160{ }^{\circ} \mathrm{C}$ via evaporation. The supernatants were repeatedly mixed with the concentrated GNPs in the base resin until the GNP content of the masterbatch reached $2 \mathrm{wt} \%$.

\subsection{Preparation of GNP/EP Nanocomposites}

The GNP content of the GNP/EP nanocomposites varied from 0 to $1 \mathrm{wt} \%$ by diluting the masterbatch with neat epoxy resin. The epoxy resin containing the masterbatch and hardener were mixed at a stoichiometrically equivalent ratio at room temperature (RT). Then, the mixture was poured into a Teflon mold and cured at $70{ }^{\circ} \mathrm{C}$ for 1 day. The sample is denoted as GEN X-YZ, where " $\mathrm{X}$ " indicates the wt \% of GNPs in the GNP/EP nanocomposite and "YZ" denotes the hardener. Sample codes and compositions of the GNP/EP nanocomposites are given in Table S1.

\subsection{Measurements}

Raman spectra were recorded with a micro-Raman spectrometer (Nanofinder 30, Tokyo, Tokyo Instruments Inc.), using a 532-nm-wavelength laser. High-resolution transmission electron microscopy was performed with a JEM 2010 system (JEOL, Akishima, Tokyo, Japan). X-ray photoelectron spectroscopy (XPS, Theta Probe AR-XPS System, Thermo Fisher Scientific, Waltham, MA, USA) was employed to analyze the composition of the GNP surface. Fourier transform infrared (FT-IR) spectra were recorded between 4000 and $400 \mathrm{~cm}^{-1}$ at a resolution of $4 \mathrm{~cm}^{-1}$ in 50 scans using an FT/IR-300E apparatus (Jasco, Easton, Easton, MD, USA). The samples were prepared by dropping the solution onto pure $\mathrm{KBr}$ pellets. A Phoenix150 apparatus (SEO, Suwon, Korea) was used to perform contact angle measurements. The static contact angles of liquids were measured by depositing a drop of 3-5 $\mu \mathrm{L}$ on the substrate, and the values were recorded as the tangent normal to the drop at the intersection between the liquid and the solid surface. The images were captured within $30 \mathrm{~s}$ of drop deposition, and the reported contact angles are the average of at least five tests performed at different locations on the 
surface. Differential scanning calorimetry (DSC) measurements were performed with a Q20 apparatus (TA INST., New Castle, DE, USA). DSC thermograms were recorded for all of the synthesized polymers, which were sealed in aluminum pans in a dry nitrogen atmosphere, with an empty aluminum pan as a reference. The scanning was performed at a rate of $10^{\circ} \mathrm{C} / \mathrm{min}$. The gel fraction and swelling ratio of the samples were determined by soaking the samples in DMAc for $48 \mathrm{~h}$ at RT. The gel fraction and swelling ratio were calculated using the following equations:

$$
\begin{gathered}
\text { Swelling ratio }=W_{s} / W_{i} \\
\text { Gel fraction }=W_{d} / W_{i} \times 100
\end{gathered}
$$

where $W_{i}$ is the weight of the sample before swelling, $W_{s}$ is the weight of the insoluble polymer in DMAc, and $W_{d}$ is the weight of the dried swollen sample at $110^{\circ} \mathrm{C}$. Dynamic mechanical analysis was performed using a Q800 apparatus (TA INST., New Castle, DE, USA). The scanning was performed at a rate of $5{ }^{\circ} \mathrm{C} / \mathrm{min}$ under a nitrogen atmosphere. Dumbbell specimens with a width of $5 \mathrm{~mm}$ and a length of $20 \mathrm{~mm}$ were obtained by curing after the casting of GNP/EP nanocomposites in a dumbbell-shaped mold. A tensile test of the composites was performed using a LLOYD LR5K PLUS instrument at a crosshead speed of $1 \mathrm{~mm} / \mathrm{min}$ at RT. Rheological experiments were performed using an AR2000 instrument (TA INST., New Castle, DE, USA), with decreasing frequency sweeps of 100-0.1 rad/s at a constant strain of $1 \%$ and various temperatures. A scanning electron microscope (SEM) (AIS2100C; Seron Tech Inc., Uiwang, Korea) was used for the scratch healing test, where a razor blade was applied to produce cuts in the middle of the films.

\section{Results and Discussion}

\subsection{Characterization of GNPs in the Masterbatch}

The quality of the fabricated GNPs is determined by the number of layers and surface defects. Raman spectroscopy and TEM imaging are useful tools for characterizing GNPs. The Raman spectra of the EG and GNPs are shown in Figure 1a. The ratio of the D to G band intensities, shown in Figure 1a, is related to the defect concentration of the GNP surface due to the overall movement of the $s p 2$ carbon atom in the basal plane $[38,39]$. The $I_{D} / I_{G}$ value for the GNPs was 0.14 , which is lower than that of the EG, the starting material employed for preparing GNPs in this study, due to the reduction via microwave irradiation. The ratio of the $2 \mathrm{D}$ to $\mathrm{G}$ band intensities is also used to evaluate the quality and thickness of the GNPs. Green and Hersam reported that as the thickness of a GNP increases, $I_{2 D} / I_{G}$ decreases from a maximum of $2.1 \pm 0.2$ for a single-layer GNP to $0.8 \pm 0.1$ for a quadruple-layer GNP [40]. The $I_{2 D} / I_{G}$ value for the GNPs was calculated to be 0.53 , corresponding to approximately 7 layers. The inset in Figure $1 \mathrm{~b}$ displays the thickness of the GNPs, confirming a GNP layer number of approximately 10, which is close to the value calculated from $I_{2 D} / I_{G}$ in the Raman spectrum. On average, the lateral size of the GNPs was $33.2 \mu \mathrm{m}$ (Figure 1c). The GNPs in the masterbatch in this study showed excellent storage stability, without any precipitation for two months.

(a)

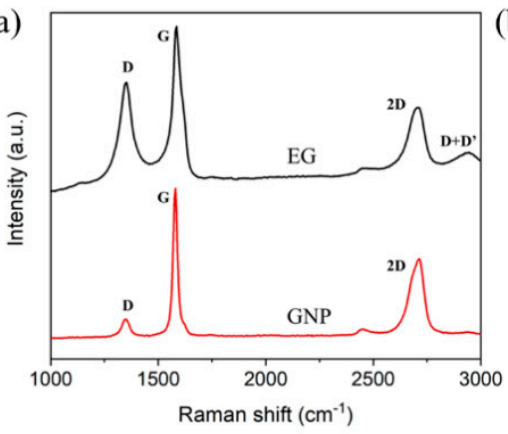

b)

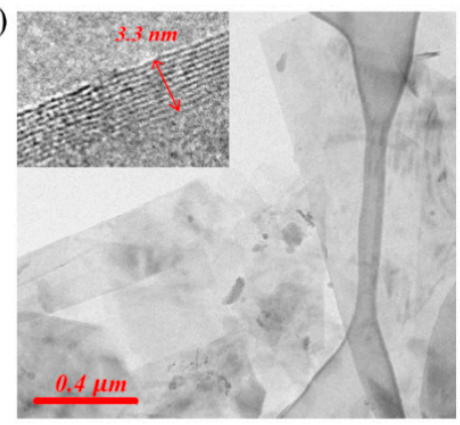

(c)

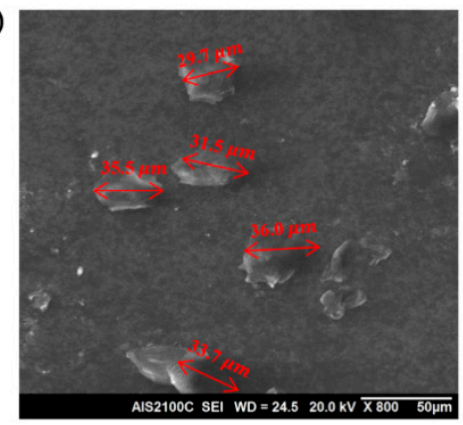

Figure 1. Raman spectra (a), HR-TEM image (b), and SEM image (c) of the graphene nanoplates (GNPs). 


\subsection{DA Reaction in the Preparation of GNP/EP Nanocomposites}

Before confirming the DA reaction in the GNP/EP nanocomposites, XPS spectra were obtained to confirm the DA reaction of the GNPs with FA. GNPs and FA were allowed to react at $70^{\circ} \mathrm{C}$ for 1 day, washed 3 times with acetone, and then dried at $70^{\circ} \mathrm{C}$ for 1 day. The obtained GNPs were designated as modified GNPs. As shown in Figure S1, both the GNPs and modified GNPs exhibited C 1s and O 1 s peaks at 284.6 and $532.5 \mathrm{eV}$, respectively [41]. A new peak at $399.5 \mathrm{eV}$ for $\mathrm{N} 1 \mathrm{~s}$ was observed in the modified GNPs, which was attributed to the DA reaction of the GNPs and FA [41]. Thus, it is postulated that the DA reaction can occur between the GNPs and FA.

Figure 2a illustrates the behavior of FA with different interfacial tensions in the mixture. The GNPs and FA can be close to each other when the relationship of interfacial tension is as follows:

$$
\gamma_{G F}<\gamma_{E F}+\gamma_{E G}
$$

where $\gamma_{E G}, \gamma_{E F}$, and $\gamma_{G F}$ are the interfacial tensions between the epoxy resin and GNPs, the epoxy resin and FA, and the GNPs and FA, respectively. When this relationship is satisfied, a DA reaction can occur between the GNPs and FA since FA will be located close to the GNP surface. In a previous study, we confirmed an in-situ DA reaction between GNPs and furfuryl derivative for nanocomposites comprising polyurethane and GNPs [42]. The surface tension values and their components can be calculated based on the relationship between the Young and Wu equations. If the contact angles of at least two liquids with known dispersion and polar components are determined for a solid surface, then $\gamma_{s}^{d}$ and $\gamma_{s}^{p}$, as well as the surface tension of the solid, can be calculated. Using these parameters, the interfacial tension between two materials can be calculated using the $\mathrm{Wu}$ or Owen-Wendt equation. The obtained surface and interfacial tension values are shown in Table S2 and Table 1. The calculated interfacial tensions for the DGEBA systems, 3.54 $\left(\gamma_{G F}\right)<5.35\left(\gamma_{E F}\right)+7.95\left(\gamma_{E G}\right)$, satisfy Equation (6), whereas those for the HDE systems, 3.54 $\left(\gamma_{G F}\right)>1.58\left(\gamma_{E F}\right)+0.57\left(\gamma_{E G}\right)$, do not satisfy Equation (6). Thus, the DA reaction can occur in the DGEBA curing process, whereas it is unlikely to occur in the HDE curing process. The FT-IR and DSC results confirm this assumption, as shown in Figure 3.

(a)

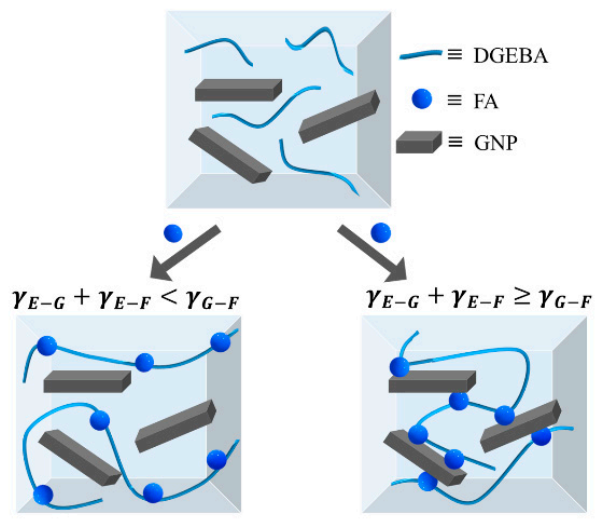

(b)

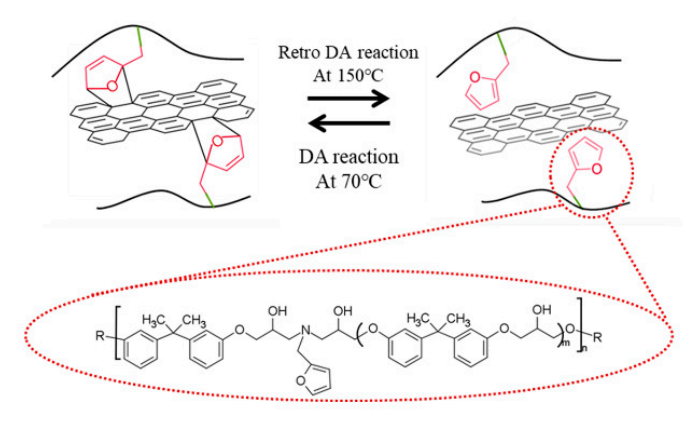

Figure 2. Schematics of two different dispersion behaviors in the system (a), and Diels-Alder (DA) and retro DA reactions between the GNPs and furfurylamine (FA) in the graphene-nanoplate/epoxy (GNP/EP) composites (b).

Table 1. Interfacial tension between the constituents of the GNP/EP nanocomposites.

\begin{tabular}{ccc}
\hline \multirow{2}{*}{ Materials } & \multicolumn{2}{c}{ Interfacial Tension (dyne/cm) } \\
\cline { 2 - 3 } & Wu & Owen-Wendt \\
\hline DGEBA/GNP & 7.95 & 4.06 \\
DGEBA/FA & 5.35 & 2.79 \\
HDE/GNP & 0.57 & 0.29 \\
HDE/FA & 1.58 & 0.79 \\
GNP/FA & 3.54 & 1.79 \\
\hline
\end{tabular}


The opening reaction of the oxirane ring in DGEBA upon curing was confirmed by FT-IR spectroscopy, as shown in Figure S2. After curing, the peak intensity at $915 \mathrm{~cm}^{-1}$ due to the oxirane ring in DGEBA was substantially reduced by the reaction $[5,10,12,15,16]$. In Figure 3a, FA shows characteristic peaks at $1530-1570$ and $1339 \mathrm{~cm}^{-1}$ due to double bonds and divinyl alkyl ether bonds, respectively. After the DA reaction, the peak intensity of the furfuryl group is reduced [43]. The $C=C$ and $=\mathrm{C}-\mathrm{O}-\mathrm{C}=$ bonds in FA disappeared in GEN-0.1-D, while the peaks in GEN-0.1-HD remained. Figure $3 b$ shows the DSC thermograms of GEN-0.1-HD and GEN-0.1-HD. The endothermic peak at approximately $90-160{ }^{\circ} \mathrm{C}$ in GEN-0.1-D is attributable to the retro DA reaction [5,9-11,15-17]. Thus, it is postulated that the DA reaction occurred in the DGEBA systems but not in the HDE systems.
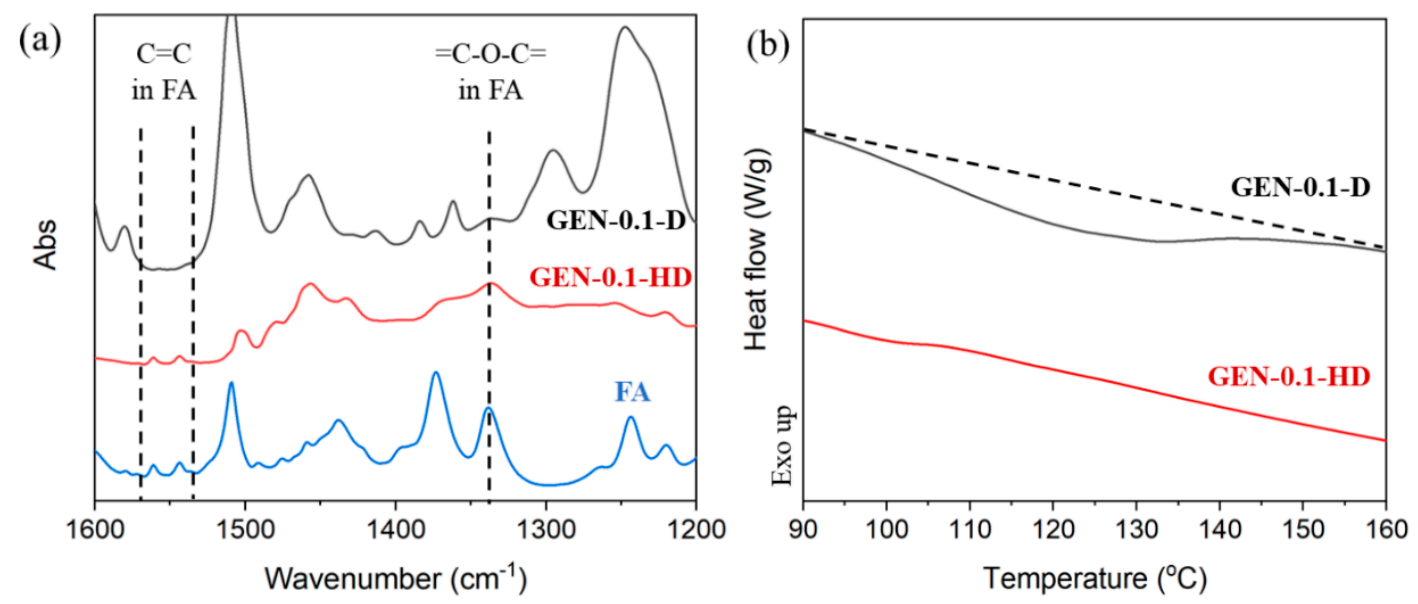

Figure 3. FT-IR spectra (a) and DSC thermograms (b) of GEN-0.1-D and GEN-0.1-HD.

Figure 4 illustrates the three types of GNP/EP nanocomposite systems investigated in this work. The DGEBA in the Diels-Alder epoxy resin (D system) can form a crosslinking structure through the DA reaction with FA when GNPs are added. Control A epoxy resin (CA system) was prepared using CA as the hardener instead of FA. CA was selected as a hardener because it can synthesize a linear backbone but does not contain diene. Hence, in the CA system, DGEBA cannot react with GNPs via the DA reaction, and the CA system can be compared with the D system. However, comparing the CA system and the D system with respect to the recycling of the crosslinked resins is not appropriate because of the linear backbone of the CA system. Therefore, control B epoxy resin (CB system) was manufactured using DETA, a commonly used crosslinking agent, as the hardener, and the CB system was compared with the D system to confirm the recyclability by the retro DA reaction.

One double bond and a dialkyl ether exist in the DA linkage formed by the GNPs and FA. Meanwhile, when the retro DA reaction occurs, a conjugated $\mathrm{C}=\mathrm{C}$ bond and a divinyl alkyl ether in FA are formed. Figure 5 shows the FT-IR spectra of GEN-0.1-DE at various temperatures. The FT-IR spectrum of GEN-0.1-DE at RT is denoted as the 1st DA. The same sample was heated to $150{ }^{\circ} \mathrm{C}$ for $30 \mathrm{~min}$. The recorded spectrum is denoted as the r-DA. Then, the r-DA sample was left at $70^{\circ} \mathrm{C}$ for 1 day. The final dataset was denoted as the 2 nd DA. The $C=C$ bond in the FA linkage $\left(1530-1570 \mathrm{~cm}^{-1}\right)$ was not observed in the 1st or 2nd DA. However, this peak was observed at an elevated temperature of $150{ }^{\circ} \mathrm{C}$. The $=\mathrm{C}-\mathrm{O}-\mathrm{C}=$ peak $\left(1339 \mathrm{~cm}^{-1}\right)$ of the 1st and 2nd DA was smaller than that of the r-DA. The asymmetric and symmetric stretching of-C-O-C-in the DA linkage $\left(1120\right.$ and $\left.863 \mathrm{~cm}^{-1}\right)$ decreased in the r-DA [44]. This change in peak intensity indicates that the DA linkage is maintained at RT and $70{ }^{\circ} \mathrm{C}$, while the reverse reaction proceeds at $150{ }^{\circ} \mathrm{C}$. Thus, it was confirmed that the DA reaction proceeded well during the epoxy curing process. 


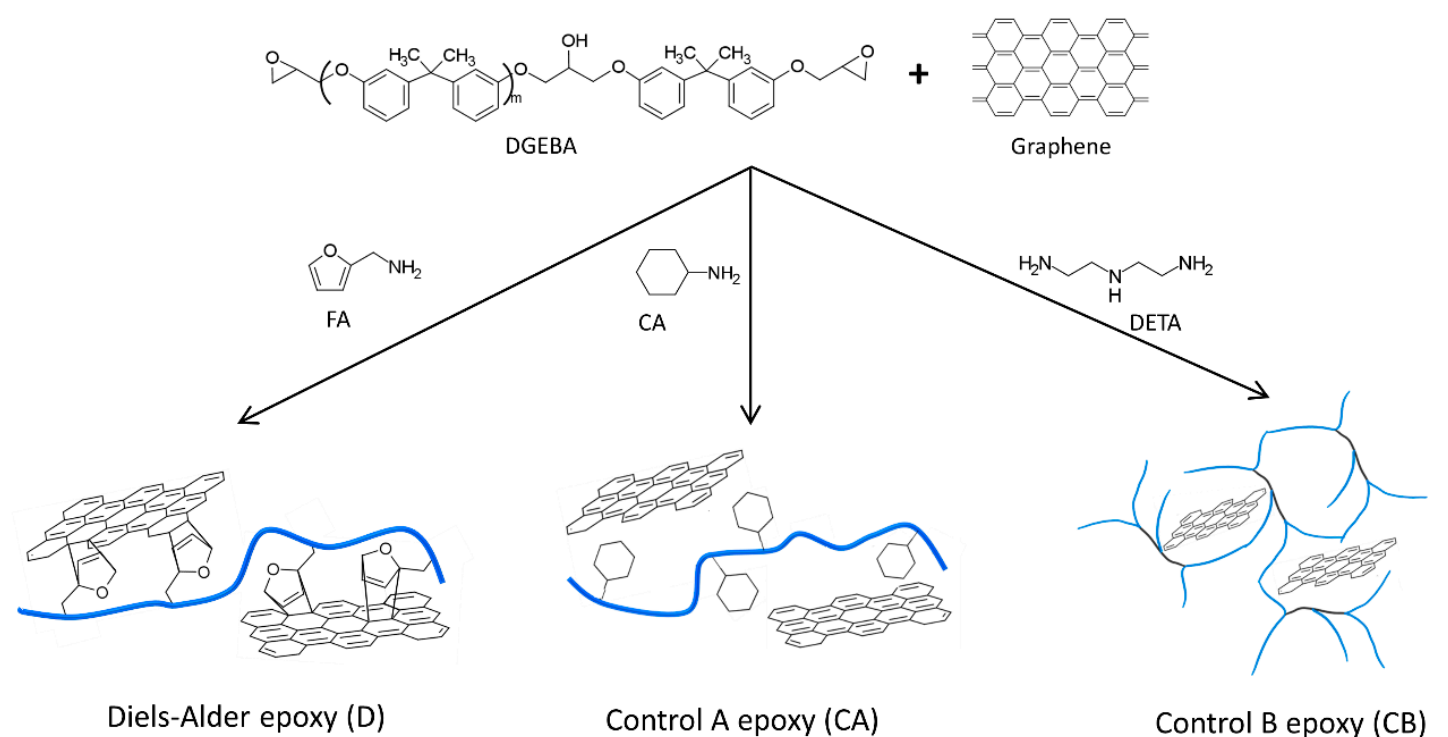

Figure 4. Schematic of the three types of GNP/EP nanocomposite systems.

(a)

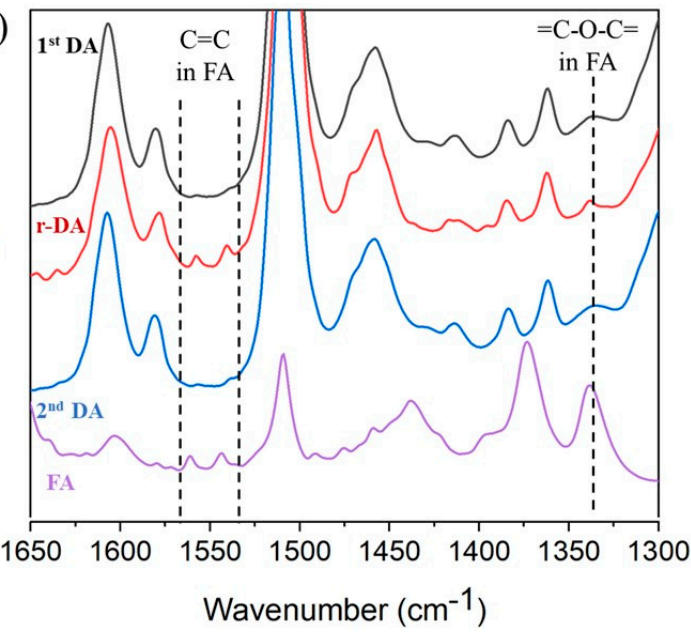

(b)

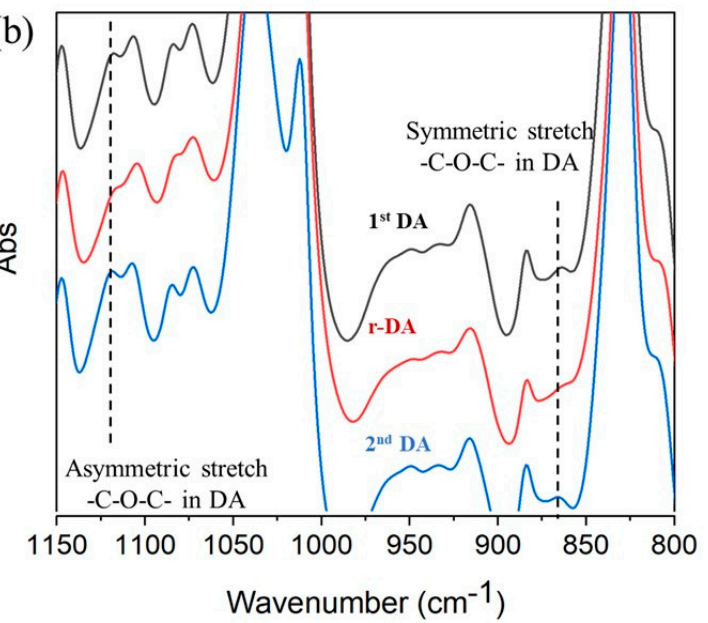

Figure 5. FT-IR spectra of GEN-0.1-D at various temperatures for wavenumbers ranging from 1650 to $1300 \mathrm{~cm}^{-1}$ (a) and 1150 to $800 \mathrm{~cm}^{-1}(\mathbf{b})$.

The endothermic peak of the DSC thermogram is often used as evidence of a retro DA reaction [5,9-11,15-17]. A secondary scan was performed using two methods to identify the endothermic peak due to the retro DA reaction. For the first secondary scan method, the sample is cooled to $0{ }^{\circ} \mathrm{C}$ immediately after the first scan, followed by a secondary scan. For the second secondary scan method, the secondary scan is performed after heat treatment in an oven at $70{ }^{\circ} \mathrm{C}$ for 1 day. The original DSC thermogram data are shown in Figure S3. Figure 6a,b presents the DSC thermograms of CA and D systems with various GNP contents. The CA system does not exhibit an endothermic peak because there was no functional group for the DA reaction. For the D systems containing GNPs, endothermic peaks due to the retro DA reaction were observed at $100 \sim 160{ }^{\circ} \mathrm{C}$ in the first and second scans after isothermal processing at $70{ }^{\circ} \mathrm{C}$ for 1 day. However, endothermic peaks due to the retro DA reaction were not observed for the quenched samples. DSC thermograms showing endotherms due to the retro DA reaction after repeated tests are shown in Figure S4. Endotherms due to the retro DA reactions were observed in the repeated tests, and thus, the reversibility of thermoset GEN-0.5-D was confirmed. 
(a)

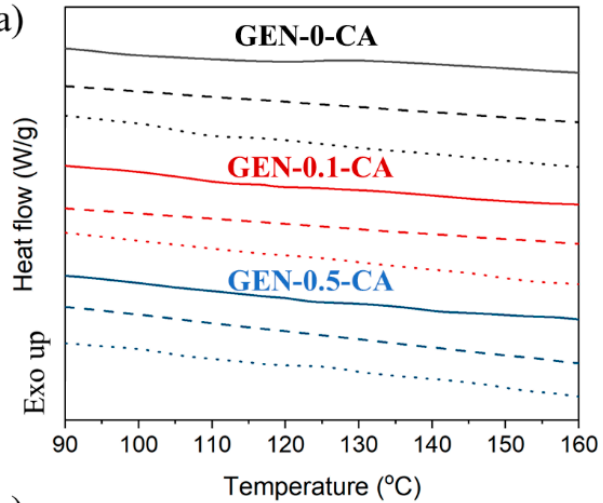

(c)

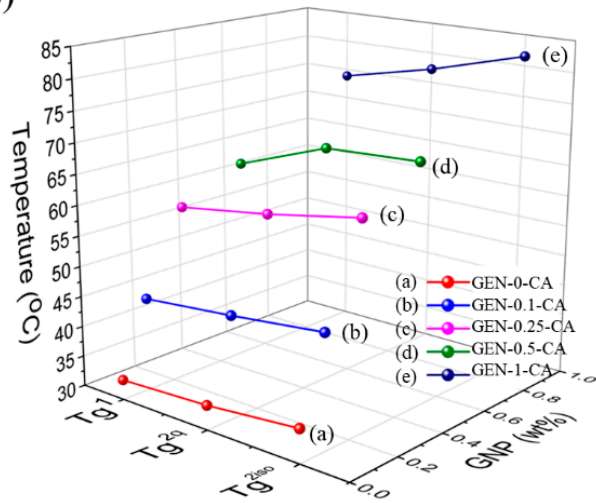

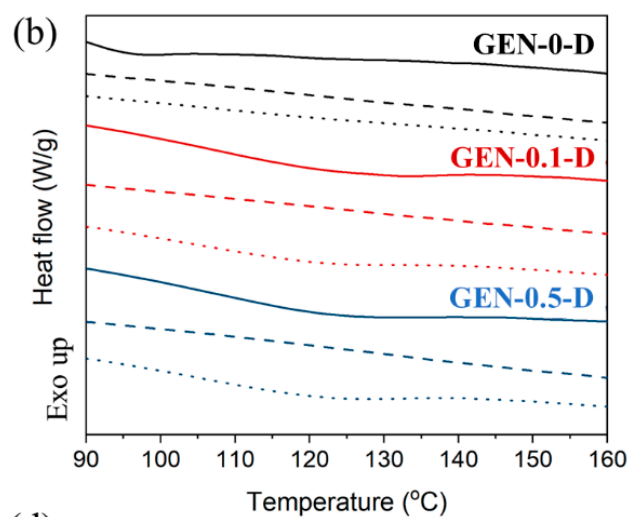

(d)

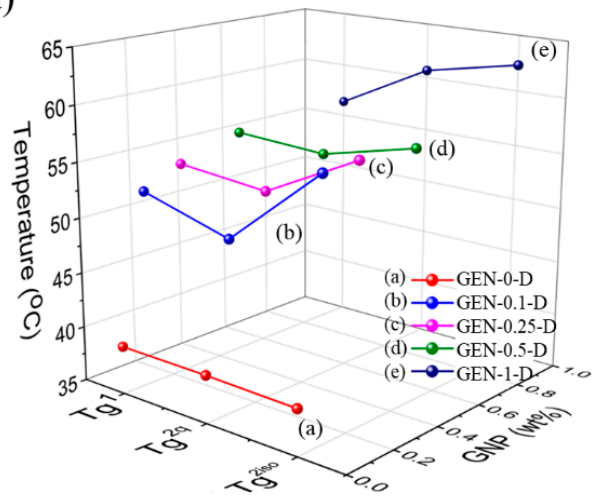

Figure 6. DSC thermogram of the control A epoxy resin (CA) and Diels-Alder epoxy resin (D systems). (a) and (b) present the heat flows of the composites (solid line: first scan, dashed line: second scan after the quenching process, dotted line: second scan after isothermal processing at $70^{\circ} \mathrm{C}$ for 1 day). (c) and (d) display the changes in $T_{\mathrm{g}}$ based on the measurement method and GNP content $\left(T_{\mathrm{g}}{ }^{1}: T_{\mathrm{g}}\right.$ for the first scan, $T_{\mathrm{g}}{ }^{2 \mathrm{q}}: T_{\mathrm{g}}$ for the second scan after the quenching process, $T_{\mathrm{g}}{ }^{2 i s o}: T_{\mathrm{g}}$ for the second scan after the isothermal process).

Therefore, it is postulated that the DA and retro DA reactions occur repeatedly in GNP/EP composites containing GNPs and FA. In Table 2, the efficiencies obtained from the enthalpy change in the endothermic peak of the D systems are given. As the GNP content increases, the efficiency of the DA reaction decreases due to the increased crosslinking density. Figure $6 c, d$ and Table 3 show the $T_{\mathrm{g}}$ changes for the CA and D systems, respectively, based on the measurement method and GNP content. The $T_{\mathrm{g}}$ value of the CA and D systems increased with increasing GNP content. Generally, in well-dispersed GNP composites, the large interfacial surface area formed by the nanofiller affects the behavior of the surrounding polymer matrix, which reduces the mobility of the polymer chain, thereby increasing $T_{\mathrm{g}}[45,46]$. Although the $T_{\mathrm{g}}{ }^{2 \mathrm{q}}$ values of the DE systems were lower than $T_{\mathrm{g}}{ }^{1}$ and $T_{\mathrm{g}}{ }^{2 \text { iso }}$, the increase in $T_{\mathrm{g}}$ in the $\mathrm{D}$ system was limited by the imparted mobility of the polymer chain due to reversible crosslinking through the retro DA reaction.

Table 2. Enthalpy changes in the GEN-X-D composites.

\begin{tabular}{cccc}
\hline Sample & 1st $\boldsymbol{\Delta} \boldsymbol{H}(\mathbf{J} / \mathbf{g})$ & 2nd $\boldsymbol{\Delta} \boldsymbol{H}(\mathbf{J} / \mathbf{g})$ & Efficiency $^{\mathbf{a}} \mathbf{( \% )}$ \\
\hline GEN-0-D & - & - & - \\
GEN-0.1-D & 3.5 & 2.4 & 68.6 \\
GEN-0.25-D & 5.7 & 3.2 & 56.4 \\
GEN-0.5-D & 6.8 & 2.9 & 41.9 \\
GEN-1-D & 4.8 & 1.5 & 31.5 \\
\hline
\end{tabular}

${ }^{\text {a }}$ Efficiency $(\%)=\left(2^{\text {nd }} \Delta H / 1^{\text {st }} \Delta H\right) \times 100$. 
Table 3. Summary of $\mathrm{Tg}$ based on the measurement method and GNP content.

\begin{tabular}{cccccccc}
\hline Sample & $\boldsymbol{T}_{\mathbf{g}}{ }^{\mathbf{1}}\left({ }^{\circ} \mathbf{C}\right)$ & $\boldsymbol{T}_{\mathbf{g}}{ }^{\mathbf{2 q}}\left({ }^{\circ} \mathbf{C}\right)$ & $\boldsymbol{T}_{\mathbf{g}}{ }^{2 \text { is }}{ }^{\circ}\left({ }^{\circ} \mathbf{C}\right)$ & Sample & $\boldsymbol{T}_{\mathbf{g}}{ }^{\mathbf{1}}\left({ }^{\circ} \mathbf{C}\right)$ & $\boldsymbol{T}_{\mathbf{g}}{ }^{2 \mathbf{q}}\left({ }^{\circ} \mathbf{C}\right)$ & $\boldsymbol{T}_{\mathbf{g}}{ }^{2 \text { iso }}\left({ }^{\circ} \mathbf{C}\right)$ \\
\hline GEN-0-CA & 33.3 & 34.0 & 35.59 & GEN-0-D & 39.4 & 39.3 & 39.1 \\
GEN-0.1-CA & 45.3 & 46.7 & 58.4 & GEN-0.1-D & 52.7 & 50.4 & 57.6 \\
GEN-0.25-CA & 58.5 & 60.7 & 63.6 & GEN-0.25-D & 54.3 & 53.5 & 57.8 \\
GEN-0.5-CA & 63.1 & 68.5 & 69.3 & GEN-0.5-D & 55.8 & 55.4 & 57.4 \\
GEN-1-CA & 73.9 & 77.2 & 81.5 & GEN-1-D & 56.4 & 60.6 & 62.3 \\
\hline
\end{tabular}

\subsection{Thermal Self-Healing Properties of GNP/EP Nanocomposites}

The D system formed a crosslinking structure through the DA reaction, which can be confirmed via a swelling test. Figure 7a shows the results of the swelling test. Nanocomposite specimens were soaked with DMAc at RT for two days. Most GEN-X-CA composites are soluble in DMAc due to its linear structure, and a gel was observed only for GEN-1-CA. The gel observed for GEN-1-CA was attributed to the crosslinking of the CA system by reactions with functional groups on the GNP surface, as confirmed by the XPS data shown in Figure S1 for the GNPs. The functional groups of GNPs in the form of carboxylic acid, hydroxyl, and epoxide have been demonstrated by XPS in previous studies [47,48], Meanwhile, all of the D system composites produced a gel. The gel content of GEN-0.5-D is $84 \%$, whereas the gel content of GEN-1D is $91 \%$. The D system nanocomposites with a higher GNP concentration have a higher crosslinking density than $\mathrm{D}$ system nanocomposites with a low GNP concentration. The swelling ratio exhibits a maximum for GEN-0.25D due to its low crosslinking density. Furthermore, the internal space in the network structure with low-crosslinking-density composites is sufficiently large to trap the solvent. For high-GNP-concentration composites, the crosslinking density is increased; hence, the internal space in which the solvent is trapped is reduced, and the swelling ratio decreases [49]. The content or swelling ratio of the gel formed by the DA reaction can be changed by the decrease in crosslinking density caused by the retro DA reaction at high temperatures. Figure $7 \mathrm{~b}$ shows that the solid specimen transforms into a fluid after the heat treatment of GEN-0.25-D in DMAc. The gel that swelled on GEN-0.25-D was completely soluble with DMAc after heat treatment at $150{ }^{\circ} \mathrm{C}$ for $1 \mathrm{~h}$, proving that the retro DA reaction occurred at $150{ }^{\circ} \mathrm{C}$.

(a)

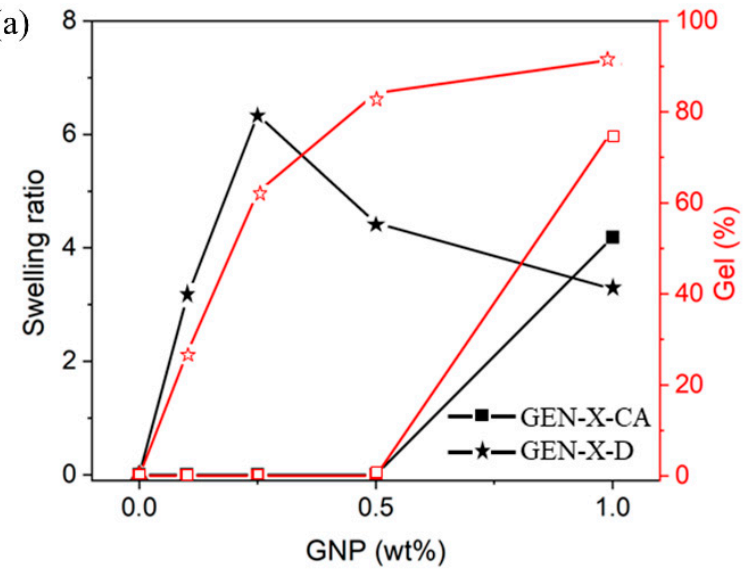

(b)

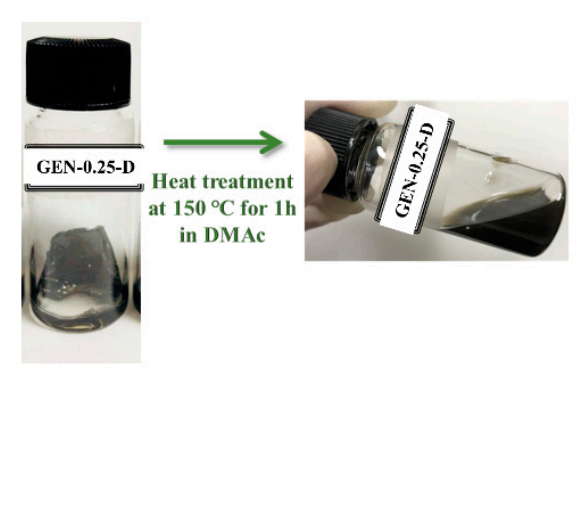

Figure 7. (a) Swelling test results for composites of the CA and D systems, (b) Digital image of GEN-0.25-D after heat treatment in DMAc.

The dynamic mechanical properties of the $\mathrm{D}$ and $\mathrm{CB}$ systems are illustrated in Figure $8 \mathrm{a}$. The storage modulus $\left(G^{\prime}\right)$ versus temperature $(T)$ curve of the nanocomposites in the CB system is typical for a crosslinked thermoset. Both composites show clear transitions from a glassy to a rubbery state, and the $G^{\prime}$ value of the D system composites in the glassy state gradually increases as the GNP content increases, due to crosslinking between the GNPs and DGEBA via the DA reaction. The composites of the D system show significantly lower glass transition temperatures in comparison with those for the 
$\mathrm{CB}$ system due to the retro DA reactions at elevated temperatures. Figure $8 \mathrm{~b}$ shows stress-strain curves for GEN-0.5-D and GEN-0.5-CB. The tensile modulus of GEN-0.5-D is larger than that of GE-0.5-CB due to crosslinking between the GNPs and the epoxy resin in the GEN-0.5-D. In the recycling tests for GEN-0.5-D, recycled specimens were successfully prepared by compression molding of powdered specimens with a hot press after the tensile test. However, powdered specimens of GEN-0.5-CB could not be remolded after the tensile test. GEN-0.5-D exhibited a tensile strength of $17.44 \mathrm{MPa}$ at an elongation of $8.51 \%$. In the recyclability test, the tensile strength of GEN-0.5-D recovered by $65 \%$ in the first cycle, and in the second cycle, a recovery of $60 \%$ was observed compared with the original GEN-0.5-D value. Water condensation of DA adducts may be one of the probable reasons for the limited rework ability after processing at $\mathrm{T}>120^{\circ} \mathrm{C}$ as reported by Cheng and Huber [50].
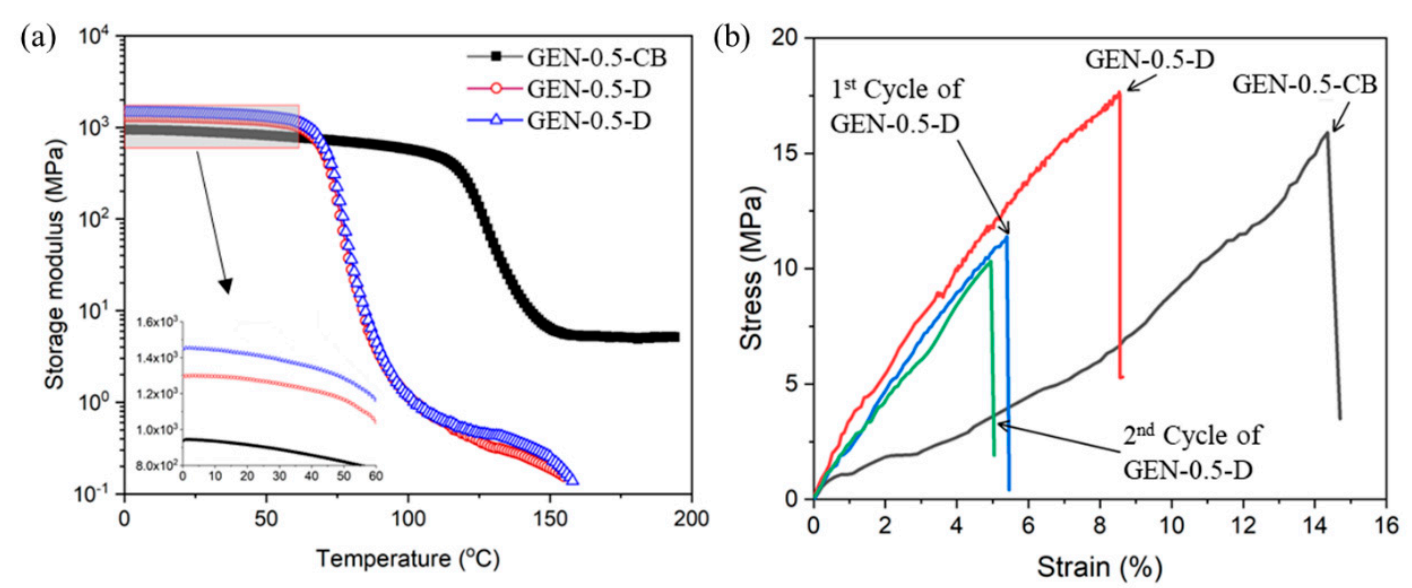

Figure 8. (a) Storage modulus of control B epoxy resin (CB) and D system composites, (b) Repeated recycling test results for GEN-0.5-CB and GEN-0.5-D.

Rheological measurements of thermosetting resins provide insights into chemical and physical changes caused by reactions occurring at specific temperatures. Figure 9 shows the changes in $\mathrm{G}^{\prime}$ and $G^{\prime \prime}$ at various temperatures over different frequency sweeps. The composites of CA systems have a loss modulus that is higher than the storage modulus regardless of the GNP content or measurement temperature. These composites show a liquid-like behavior. Meanwhile, for the D system composites, the loss modulus intersects with the storage modulus, and the composite can flow beyond the gel point. The crossover of $\mathrm{G}^{\prime}$ and $\mathrm{G}^{\prime \prime}$ occurs at 0.20 and $50.12 \mathrm{rad} / \mathrm{s}$ for 80 and $120^{\circ} \mathrm{C}$ in GEN-0.1-D, with fluidity occurring at $150{ }^{\circ} \mathrm{C}$. The relaxation times obtained from the frequency values for the crossover of $\mathrm{G}^{\prime}$ and G" are given in Table S3. In general, the curing process of the thermoset resin caused the polymer to exhibit glassy or liquid-like behavior based on the gel point. Considering that the crosslinking of the DA reaction is due to a dynamic covalent bond, the breakdown and reforming of the structure over different timescales can be identified by the temperature-dependent behavior [51].

A scratch on the surface of the polymer specimens can be healed when reversible or exchange reactions are introduced in the polymer. The specimens were scratched with a razor blade and then gold-coated. The thermal self-healing properties of the nanocomposites were investigated after heat treatment at $150{ }^{\circ} \mathrm{C}$ on a heating plate for $1 \mathrm{~h}$. Figure 10a shows representative SEM images of the scratched specimens before and after the self-healing tests. The scratch remained in GEN-0.5-CB, while that of GEN-0.5-D gradually disappeared within $1 \mathrm{~h}$. The scratch healing ability was imparted to GEN-0.5-D by the retro DA reactions. We infer that the small pieces can be reprocessed at elevated temperatures via the retro DA reaction. To confirm the reprocessing ability, GEN-0.5-CB and GEN-0.5-D were pulverized, and then the samples were placed between two steel plates and heat treated at $160^{\circ} \mathrm{C}$ for $0.5 \mathrm{~h}$ with an external force of less than $3 \mathrm{MPa}$. Figure $10 \mathrm{~b}$ shows the reprocessed samples before and after reprocessing. The pieces of GEN-0.5-CB remain after the heat treatment; however, a film 
was obtained from the pulverized GEN-0.5-D sample after heat treatment under the same conditions employed for GEN-0.5-CB.
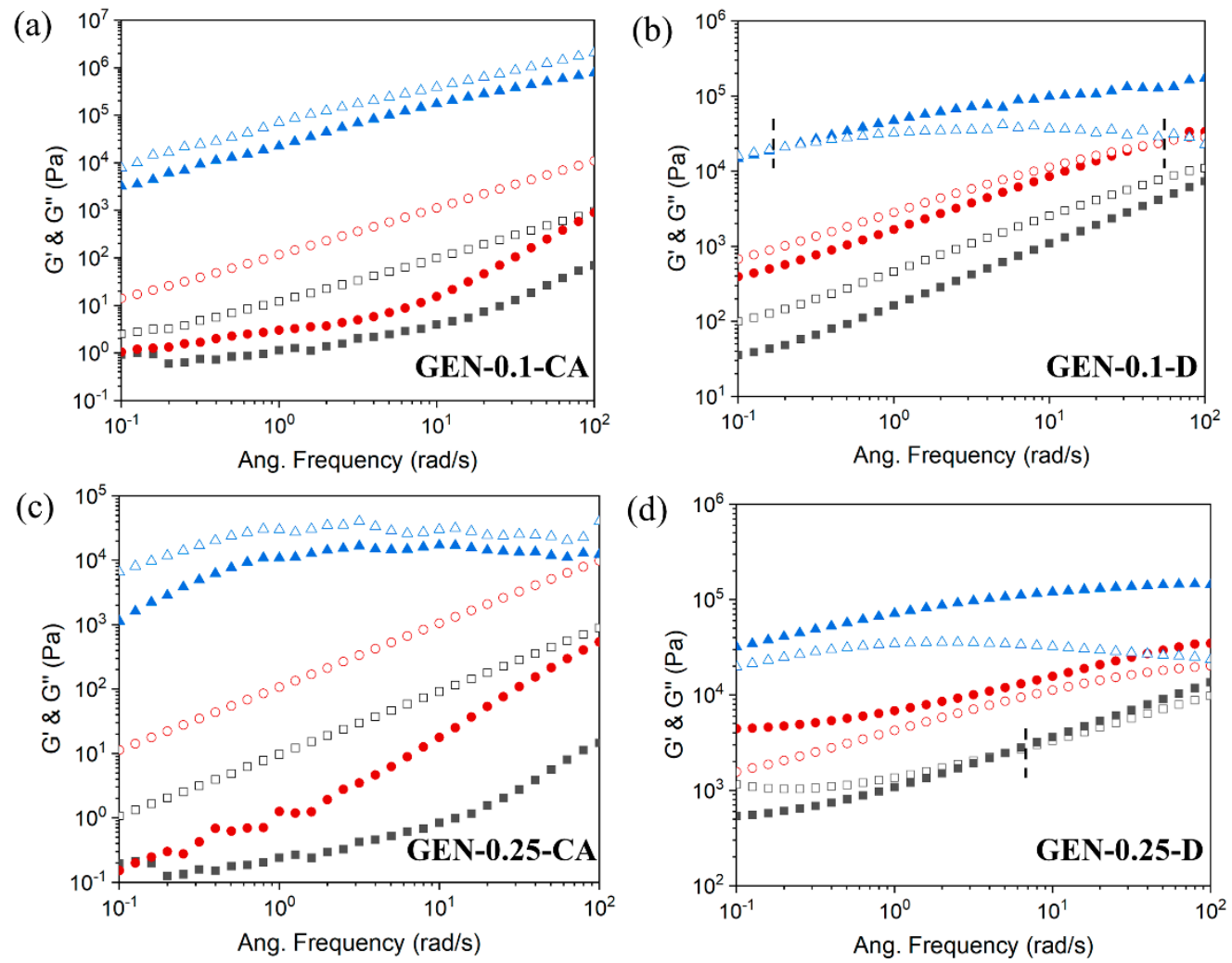

Figure 9. Storage (filled symbols) and loss (open symbols) moduli for composites of the CA and D systems at $80^{\circ} \mathrm{C}(\boldsymbol{\Delta}), 120^{\circ} \mathrm{C}(\bullet)$, and $150^{\circ} \mathrm{C}(\boldsymbol{\square})$.
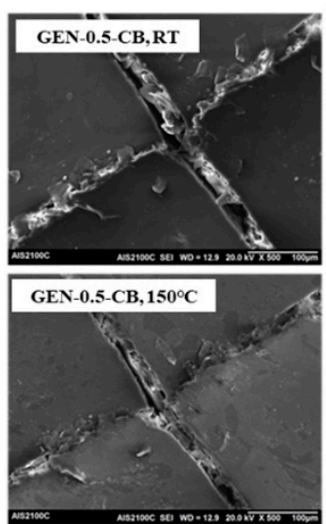

(a)

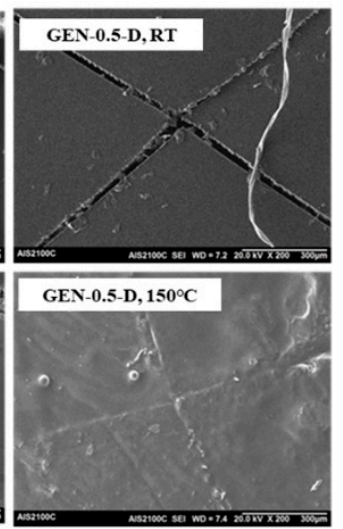

(a)

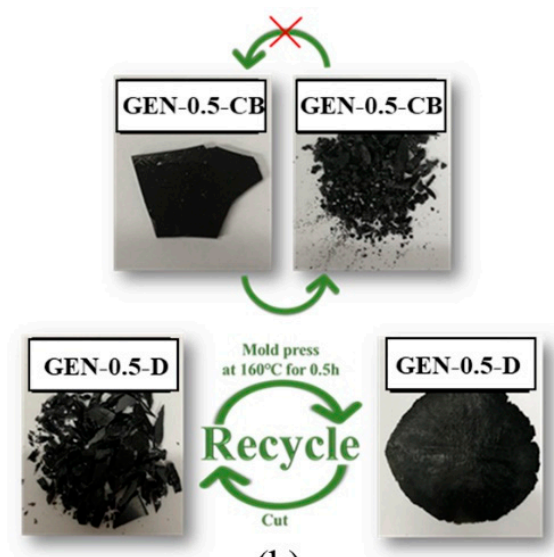

(b)

Figure 10. (a) SEM images of the scratch healing test for GEN-0.5-CB and GEN-0.5-D, and (b) reprocessing of the composites in small pieces with an external force of less than $3 \mathrm{MPa}$ and heat treatment at $160{ }^{\circ} \mathrm{C}$ for $0.5 \mathrm{~h}$.

\section{Conclusions}

In this study, thermally remendable GNP/EP nanocomposites were successfully synthesized via an in-situ DA reaction between GNPs and FA during the epoxy curing process. The interfacial tension between each material was calculated, and the conditions for the DA reaction between the GNPs and FA during the DGEBA curing process were determined. The DA and retro DA reactions were confirmed through FT-IR spectroscopy, DSC thermography, and rheological measurements. Due to the 
reversible crosslinked structure in this system, scratch healing and reprocessing ability were achieved at $150{ }^{\circ} \mathrm{C}$. The crosslinked structure of $\mathrm{D}$ system composites was exhibited at RT; however, thermal molding for recycling was possible at high temperatures due to the reversibility of the DA reaction.

Supplementary Materials: The following are available online at http://www.mdpi.com/2073-4360/11/6/1057/s1. Table S1: Sample code and compositions of the GNP/EP nanocomposites; Table S2: Components of the surface tensions $\left(20^{\circ} \mathrm{C}\right)$; Table S3: Relaxation times of D system nanocomposites; Figure S1: XPS spectra of GNPs and modified GNPs; Figure S2: FT-IR spectra of GEN-0.1-D and DGEBA; Figure S3: DSC thermograms of the CA and D systems of different GNP contents: (a) First scan; (b) Second scan after quenching at $0{ }^{\circ} \mathrm{C}$; (c) Second scan after the isothermal condition at $70^{\circ} \mathrm{C}$ for $1 \mathrm{~h}$; Figure S4: DSC thermograms of the D system composites: (a) third scan; (b) repeated endothermic peak of GEN-0.5-D.

Author Contributions: Conceptualization: D.-S.L.; Methodology: C.-R.O. and D.-I.L.; Validation: D.-S.L. and J.-H.P.; Writing —original draft preparation: C.-R.O.; Writing—review and editing: D.-S.L.; Project administration: D.-S.L.; Funding acquisition: D.-S.L.

Funding: It is acknowledged that this work was supported by the ATC project of KEIT funded by the Ministry of Trade, Industry, and Energy (Project No: ATC-10048672) and the Ministry of Trade, Industry \& Energy (MOTIE, Korea) under the Industrial Technology Innovation Program. (No. 10049677, Bio-Isosynatates and Alternative Biobased Materials for Polyurethane Using Green Carbon) in Korea.

Conflicts of Interest: The authors declare no conflicts of interest.

\section{References}

1. Liu, Y.L.; Chuo, T.W. Self-healing polymers based on thermally reversible Diels-Alder chemistry. Polymer Chem. 2013, 4, 2194-2205. [CrossRef]

2. Canadell, J.; Goossens, H.; Klumperman, B. Self-Healing Materials Based on Disulfide Links. Macromolecules 2011, 44, 2536-2541. [CrossRef]

3. Chao, A.; Negulescu, I.; Zhang, D. Dynamic Covalent Polymer Networks Based on Degenerative Imine Bond Exchange: Tuning the Malleability and Self-Healing Properties by Solvent. Macromolecules 2016, 49, 6277-6284. [CrossRef]

4. Capelot, M.; Unterlass, M.M.; Tournilhac, F.; Leibler, L. Catalytic Control of the Vitrimer Glass Transition. ACS Macro Lett. 2012, 1, 789-792. [CrossRef]

5. Kuang, X.; Liu, G.; Dong, X.; Wang, D. Triple-Shape Memory Epoxy Based on Diels-Alder Adduct Molecular Switch. Polymer 2016, 84, 1-9. [CrossRef]

6. Turkenburg, D.H.; Fischer, H.R. Diels-Alder Based, Thermo-Reversible Cross-Linked Epoxies for Use in Self-Healing Composites. Polymer 2015, 79, 187-194. [CrossRef]

7. Klein, R.; Übel, F.; Frey, H. Maleimide Glycidyl Ether: A Bifunctional Monomer for Orthogonal Cationic and Radical Polymerizations. Macromol. Rapid Commun. 2015, 36, 1822-1828. [CrossRef] [PubMed]

8. Mcelhanon, J.R.; Russick, E.M.; Wheeler, D.R.; Loy, D.A.; Aubert, J.H. Removable Foams Based on an Epoxy Resin Incorporating Reversible Diels-Alder Adducts. J. Appl. Polym. Sci. 2002, 85, 1496-1502. [CrossRef]

9. Bai, N.; Saito, K.; Simon, G.P. Synthesis of a Diamine Cross-Linker Containing Diels-Alder Adducts to Produce Self-Healing Thermosetting Epoxy Polymer from a Widely Used Epoxy Monomer. Polym. Chem. 2013, 4, 724-730. [CrossRef]

10. Kuang, X.; Liu, G.; Dong, X.; Liu, X.; Xu, J.; Wang, D. Facile Fabrication of Fast Recyclable and Multiple Self-Healing Epoxy Materials through Diels-Alder Adduct Cross-Linker. J. Polym. Sci. Part A Polym. Chem. 2015, 53, 2094-2103. [CrossRef]

11. Li, J.; Zhang, G.; Deng, L.; Jiang, K.; Zhao, S.; Gao, Y.; Sun, R.; Wong, C. Thermally Reversible and Self-Healing Novolac Epoxy Resins Based on Diels-Alder Chemistry. J. Appl. Polym. Sci. 2015, 132. [CrossRef]

12. Marref, M.; Mignard, N.; Jegat, C.; Taha, M.; Belbachir, M.; Meghabar, R. Epoxy-Amine Based Thermoresponsive Networks Designed by Diels-Alder Reactions. Polym. Int. 2013, 62, 87-98. [CrossRef]

13. Pratama, P.A.; Sharifi, M.; Peterson, A.M.; Palmese, G.R. Room Temperature Self-Healing Thermoset Based on the Diels-Alder Reaction. ACS Appl. Mater. Interfaces 2013, 5, 12425-12431. [CrossRef] [PubMed]

14. Zhang, W.; Duchet, J.; Gérard, J.F. Effect of Epoxy Matrix Architecture on the Self-Healing Ability of Thermo-Reversible Interfaces Based on Diels-Alder Reactions: Demonstration on a Carbon Fiber/Epoxy Microcomposite. RSC Adv. 2016, 6, 114235-114243. [CrossRef] 
15. Li, Q.T.; Jiang, M.J.; Wu, G.; Chen, L.; Chen, S.C.; Cao, Y.X.; Wang, Y.Z. Photothermal Conversion Triggered Precisely Targeted Healing of Epoxy Resin Based on Thermoreversible Diels-Alder Network and Amino-Functionalized Carbon Nanotubes. ACS Appl. Mater. Interfaces 2017, 9, 20797-20807. [CrossRef] [PubMed]

16. Karami, Z.; Zohuriaan-Mehr, M.J.; Rostami, A. Biobased Diels-Alder Engineered Network from Furfuryl Alcohol and Epoxy Resin: Preparation and Mechano-Physical Characteristics. Chem. Select 2018, 3, 40-46. [CrossRef]

17. Duval, A.; Couture, G.; Caillol, S.; Avérous, L. Biobased and Aromatic Reversible Thermoset Networks from Condensed Tannins via the Diels-Alder Reaction. ACS Sustain. Chem. Eng. 2017, 5, 1199-1207. [CrossRef]

18. Coleman, J.N.; Lotya, M.; O’Neill, A.; Bergin, S.D.; King, P.J.; Khan, U.; Young, K.; Gaucher, A.; De, S.; Smith, R.J.; et al. Two-Dimensional Nanosheets Produced by Liquid Exfoliation of Layered Materials. Science 2011, 331, 568-571. [CrossRef] [PubMed]

19. Paton, K.R.; Varrla, E.; Backes, C.; Smith, R.J.; Khan, U.; O’Neill, A.; Boland, C.; Lotya, M.; Istrate, O.M.; King, P.; et al. Scalable Production of Large Quantities of Defect-Free Few-Layer Graphene by Shear Exfoliation in Liquids. Nat. Mater. 2014, 13, 624-630. [CrossRef]

20. Sinha Ray, S.; Okamoto, M. Polymer/Layered Silicate Nanocomposites: A Review from Preparation to Processing. Prog. Polym. Sci. 2003, 28, 1539-1641. [CrossRef]

21. Wu, Q.; Xu, Y.; Yao, Z.; Liu, A.; Shi, G. Supercapacitors Based on Flexible Graphene/Polyaniline Nanofiber Composite Films. ACS Nano 2010, 4, 1963-1970. [CrossRef] [PubMed]

22. Araya-Hermosilla, R.; Pucci, A.; Araya-Hermosilla, E.; Pescarmona, P.P.; Raffa, P.; Polgar, L.M.; Moreno-Villoslada, I.; Flores, M.; Fortunato, G.; Broekhuis, A.A.; et al. An easy synthetic way to exfoliate and stabilize MWCNTs in a thermoplastic pyrrole-containing matrix assisted by hydrogen bonds. RSC Adv. 2016, 6, 85829-85837. [CrossRef]

23. Tang, Z.; Liu, Y.; Guo, B.; Zhang, L. Malleable, mechanically strong, and adaptive elastomers enabled by interfacial exchangeable bonds. Macromolecules 2017, 50, 7584-7592. [CrossRef]

24. Sarkar, S.; Bekyarova, E.; Niyogi, S.; Haddon, R.C. Diels-Alder Chemistry of Graphite and Graphene: Graphene as Diene and Dienophile. J. Am. Chem. Soc. 2011, 133, 3324-3327. [CrossRef] [PubMed]

25. Seo, J.-M.; Jeon, I.-Y.; Baek, J.-B. Mechanochemically Driven Solid-State Diels-Alder Reaction of Grpahite into Graphene Nanoplatelets. Chem. Sci. 2013, 4, 4273-4277. [CrossRef]

26. Ji, Z.; Chen, J.; Huang, L.; Shi, G. High-Yield Production of Highly Conductive Graphene via Reversible Covalent Chemistry. Chem. Commun. 2015, 51, 2806-2809. [CrossRef]

27. Chang, C.M.; Liu, Y.L. Functionalization of Multi-Walled Carbon Nanotubes with Furan and Maleimide Compounds through Diels-Alder Cycloaddition. Carbon N. Y. 2009, 47, 3041-3049. [CrossRef]

28. Le, C.M.Q.; Cao, X.T.; Lim, K.T. Ultrasound-Promoted Direct Functionalization of Multi-Walled Carbon Nanotubes in Water via Diels-Alder "Click Chemistry". Ultrason. Sonochem. 2017, 39, 321-329. [CrossRef]

29. Bai, J.; He, Q.; Shi, Z.; Tian, M.; Xu, H.; Ma, X.; Yin, J. Self-Assembled Elastomer Nanocomposites Utilizing C60and Poly(Styrene-b-Butadiene-b-Styrene) via Thermally Reversible Diels-Alder Reaction with Self-Healing and Remolding Abilities. Polymer 2017, 116, 268-277. [CrossRef]

30. Kötteritzsch, J.; Geitner, R.; Ahner, J.; Abend, M.; Zechel, S.; Vitz, J.; Hoeppener, S.; Dietzek, B.; Schmitt, M.; Popp, J.; et al. Remendable Polymers via Reversible Diels-Alder Cycloaddition of Anthracene-Containing Copolymers with Fullerenes. J. Appl. Polym. Sci. 2018, 135, 1-14. [CrossRef]

31. Pokharel, P.; Lee, D.S. High Performance Polyurethane Nanocomposite Films Prepared from a Masterbatch of Graphene Oxide in Polyether Polyol. Chem. Eng. J. 2014, 253, 356-365. [CrossRef]

32. Lee, S.-H.; Oh, C.-R.; Lee, D.-S. Large Improvement in the Mechanical Properties of Polyurethane Nanocomposites Based on a Highly Concentrated Graphite Nanoplate/Polyol Masterbatch. Nanomaterials 2019, 9, 389. [CrossRef] [PubMed]

33. Fowkes, F.M. Dispersion Force Contributions to Surface and Interfacial Tensions, Contact Angles, and Heats of Immersion. Contact Angle Wettability Adhes 1964, 43, 99-111.

34. Owens, D.K.; Wendt, R.C. Estimation of the Surface Free Energy of Polymers. J. Appl. Polym. Sci. 1969, 13, 1741-1747. [CrossRef]

35. Wu, S. Calculation of Interfacial Tension in Polymer Systems. J. Polym. Sci. Part C Polym. Symp. 2007, 34, 19-30. [CrossRef] 
36. Chen, J.; Wu, D. Poly (Trimethylene Terephthalate)/Poly (Butylenes Succinate) Blend: Phase Behavior and Mechanical Property Control Using Its Transesterification System as the Compatibilizer. Mater. Chem. Phys. 2014, 148, 554-561. [CrossRef]

37. Khan, U.; O’Neill, A.; Porwal, H.; May, P.; Nawaz, K.; Coleman, J.N. Size Selection of Dispersed, Exfoliated Graphene Flakes by Controlled Centrifugation. Carbon N. Y. 2012, 50, 470-475. [CrossRef]

38. Canado, L.G.; Takai, K.; Enoki, T.; Endo, M.; Kim, Y.A.; Mizusaki, H.; Jorio, A.; Coelho, L.N.; Magalhães-Paniago, R.; Pimenta, M.A. General Equation for the Determination of the Crystallite Size La of Nanographite by Raman Spectroscopy. Appl. Phys. Lett. 2006, 88, 2-5.

39. Ferrari, A.C. Raman Spectroscopy of Graphene and Graphite: Disorder, Electron-Phonon Coupling, Doping and Nonadiabatic Effects. Solid State Commun. 2007, 143, 47-57. [CrossRef]

40. Green, A.A.; Hersam, M.C. Solution Phase Production of Graphene with Controlled Thickness via Density Differentiation. Nano Lett. 2009, 9, 4031-4036. [CrossRef]

41. Lai, L.; Chen, L.; Zhan, D.; Sun, L.; Liu, J.; Lim, S.H.; Poh, C.K.; Shen, Z.; Lin, J. One-step synthesis of $\mathrm{NH} 2$-graphene from in situ graphene-oxide reduction and its improved electrochemical properties. Carbon 2011, 49, 3250-3257. [CrossRef]

42. Oh, C.-R.; Lee, S.-H.; Park, J.-H.; Lee, D.-S. Thermally Self-Healing Graphene-Nanoplate/Polyurethane Nanocomposites via Diels-Alder Reaction through a One-Shot Process. Nanomaterials 2019, 9, 434. [CrossRef] [PubMed]

43. Lee, H.Y.; Cha, S.H. Enhancement of Self-Healing Property by Introducing Ethylene Glycol Group into Thermally Reversible Diels-Alder Reaction Based Self-Healable Materials. Macromol. Res. 2017, 25, 640-647. [CrossRef]

44. Bai, J.; Shi, Z. Dynamically Cross-Linked Elastomer Hybrids with Light-Induced Rapid and Efficient Self-Healing Ability and Reprogrammable Shape Memory Behavior. ACS Appl. Mater. Interfaces 2017, 9, 27213-27222. [CrossRef] [PubMed]

45. Yu, B.; Shi, Y.; Yuan, B.; Qiu, S.; Xing, W.; Hu, W.; Song, L.; Lo, S.; Hu, Y. Enhanced Thermal and Flame Retardant Properties of Flame-Retardant-Wrapped Graphene/Epoxy Resin Nanocomposites. J. Mater. Chem. A 2015, 3, 8034-8044. [CrossRef]

46. Tang, L.C.; Wan, Y.J.; Yan, D.; Pei, Y.B.; Zhao, L.; Li, Y.B.; Wu, L.B.; Jiang, J.X.; Lai, G.Q. The Effect of Graphene Dispersion on the Mechanical Properties of Graphene/Epoxy Composites. Carbon N. Y. 2013, 60, $16-27$. [CrossRef]

47. Chen, Z.; Lu, H. Constructing Sacrificial Bonds and Hidden Lengths for Ductile Graphene/Polyurethane Elastomers with Improved Strength and Toughness. J. Mater. Chem. 2012, 22, 12479-12490. [CrossRef]

48. Stankovich, S.; Dikin, D.A.; Piner, R.D.; Kohlhaas, K.A.; Kleinhammes, A.; Jia, Y.; Wu, Y.; Nguyen, S.T.; Ruoff, R.S. Synthesis of Graphene-Based Nanosheets via Chemical Reduction of Exfoliated Graphite Oxide. Carbon N. Y. 2007, 45, 1558-1565. [CrossRef]

49. Defize, T.; Thomassin, J.M.; Alexandre, M.; Gilbert, B.; Riva, R.; Jérôme, C. Comprehensive Study of the Thermo-Reversibility of Diels-Alder Based PCL Polymer Networks. Polymer 2016, 84, 234-242. [CrossRef]

50. Cheng, Y.T.; Huber, G.W. Production of targeted aromatics by using Diels-Alder classes of reactions with furans and olefins over ZSM-5. Green Chem. 2012, 14, 3114-3125. [CrossRef]

51. Adzima, B.J.; Aguirre, H.A.; Kloxin, C.J.; Scott, T.F.; Bowman, C.N. Rheological and Chemical Analysis of Reverse Gelation in a Covalently Cross-Linked Diels-Alder Polymer Network. Macromolecules 2008, 41, 9112-9117. [CrossRef] [PubMed]

(C) 2019 by the authors. Licensee MDPI, Basel, Switzerland. This article is an open access article distributed under the terms and conditions of the Creative Commons Attribution (CC BY) license (http://creativecommons.org/licenses/by/4.0/). 